\title{
Bosques en el Perú: importancia, gestión y perspectivas en el contexto del cambio climático
}

\section{RESUMEN}

\begin{abstract}
El presente trabajo pretende presentar un panorama escueto pero integral de la situación de los bosques amazónicos peruanos. Esperando lograrlo, el primer ítem titulado: «La Amazonía para el Mundo, la Región y el Perú», hace un acercamiento de las diversas funciones que cumple esta cuenca en esos tres niveles; el segundo ítem: «La Gestión Nacional de los Bosques Amazónicos», busca acercarnos al estado de la gestión estatal de los bosques, para lo cual, incluye un sub-ítem "Deforestación», que trata de abarcar esta compleja problemática; el tercer ítem: «Perspectivas y Recomendaciones para la Gestión de los Bosques en el Contexto de Cambio Climático", trata básicamente de los diversos aspectos a considerar, en el contexto nacional, para una futura conservación de los bosques amazónicos; y finalmente, hay un par de conclusiones globales.
\end{abstract}

Palabras clave: Amazonia, bosques, Educación, Clima, Perú

\section{Forests in Peru: importance, management and perspectives in a climate change context}

\begin{abstract}
This written work intends to submit a brief but comprehensive panorama of the Peruvian Amazonian forests situation. Hoping to achieve it, the first item entitled: "The Amazon for the World, the Region and Peru", makes an approach of the diverse functions that this basin fulfills in these three levels; the second item: "The Amazonian Forests National Management", seeks to bring us closer to the state of forest management, including a sub-item "Deforestation", which pursues to encompass this complex problem; the third item: "Perspectives and Recommendations for Forests Management in a Climate Change context", basically deals with various aspects to be considered, in a national context, for a future conservation of the Amazonian forests; and finally, there are a couple of global conclusions.
\end{abstract}

KeYwords: Amazon, forests, Education, Climate Change, Peru. 


\section{Introducción}

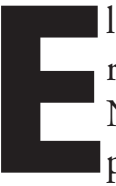

Perú presenta siete de las nueve características reconocidas por la Convención Marco de la Naciones Unidas sobre el Cambio Climático, para calificar como un país particularmente vulnerable; también cuenta con una alta exposición a amenazas de origen hidrometereológico, por lo cual, del total de emergencias nacionales, el $72 \%$ son de esta índole (República del Perú, 2015). Por otro lado, su economía está basada en el extractivismo de materias primas, dónde el 7,5\% del PBI está compuesto por actividades sensibles a los cambios en el clima (SINIA, Cifras Ambientales, 2014).

Una geografía especialmente susceptible al cambio climático, sumada a deficiencias estructurales que venimos arrastrando desde hace varios gobiernos atrás, como: la débil gobernabilidad, la limitada infraestructura, la dependencia al extractivismo y la presencia - aún significativa- de la pobreza socioeconómica, no es una buena combinación para ningún país, y menos para uno en vías de desarrollo.

Se prevé que para el 2030, se alcanzaría un PBI menor en $5,7 \%$ a $6,8 \%$ del que se alcanzaría sin cambio climático, mientras que para el 2050 la brecha respecto del PBI potencial estaría entre el 20,2\% y el 23,4\% del PBI real (República del Perú, 2015).

Entre el cambio climático y la deforestación hay una relación particular ya que son directamente interdependientes: a mayor deforestación, mayor cambio climático, y viceversa.

No existen suficientes informes o estudios precisos que se enfoquen en las consecuencias directas e indirectas que acarreará el cambio climático para el país. Los posibles impactos que se vislumbran para Perú, se conocen gracias a informes generales de organismos internacionales, pero la investigación sobre este tema, enfocada en país, es demasiado incipiente aún y si la que hay, no está debidamente sistematizada, ni interconectada. Recién en las últimas elecciones, ha habido algunos candidatos presidenciales han puesto el tema de la gestión ambiental y del cambio climático en sus planes de gobierno.

Por el lado de la sociedad civil, si bien las protestas por el manejo de los recursos naturales vienen desde ya un periodo significativo, el conocimiento del cambio climático, sus causales y efectos, cómo adaptarse y cómo mitigarlo, aún es vago o ausente, salvo en algunos ámbitos académicos. En resumen: hay mucho por hacer.

\section{Cuerpo}

\section{La Amazonía para el mundo, la región y el Perú}

Los países de la cuenca amazónica (Brasil, Colombia, Ecuador, Venezuela y Perú) están dentro de los 17 países mega diversos del planeta, principalmente, porque sus bosques primarios son los hábitats más ricos del planeta (Cordero, 2011: 5). Los bosques almacenan carbono en su biomasa (madera, hojas y suelo) y casi el 50\% del carbono secuestrado por los bosques del mundo, lo almacenan los bosques de América del Sur (Cordero, 2011: 6).

Por otro lado, la seguridad hídrica de la Amazonía depende principalmente de los servicios ecosistémicos ofrecidos por el bosque, como: reciclaje de agua de lluvia, la regulación y purificación hídrica y otros servicios vitales. El bosque recicla entre el 20-25\% de las lluvias que recibe y el aire que viaja por el bosque puede generar el doble de lluvia que el aire que viaja por la tierra deforestada (Mardas et al., 2013: 9).

CuAdro 1: Distribución NACiONAL DEL RECURSO HídRICO (SINIA, Cifras ambientales 2014)

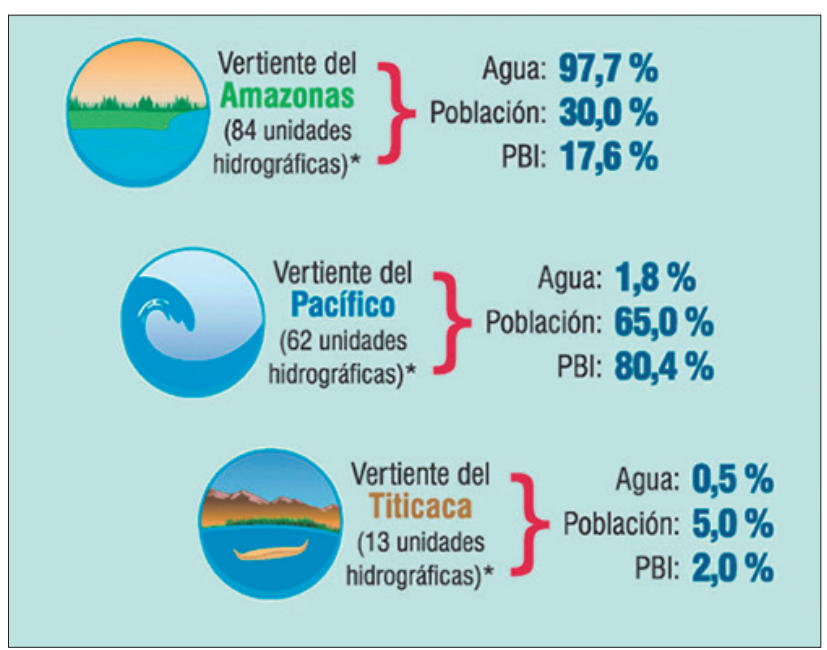

Como vemos, la Amazonía sustenta la seguridad hídrica nacional como la regional, y existe una interdependencia no solo entre bosques saludables y seguridad hídrica sino también entre seguridad hídrica, energética, alimentaria y de la salud (recursos medicinales, regulación de epidemias como la malaria, la leishmaniosis y otras enfermedades infecciosas comunes de la región). Además la inseguridad hídrica por contaminación impacta de manera especial en las poblaciones locales, ya que sólo el 55\% de los peruanos 
CUAdro 2: INTERDEPENDENCIA ENTRE LA SEGURIDAD HÍDRICA, LA ENERGÉTICA, LA ALIMENTARIA Y LA DE la SAlud en la Amazonía (Mardas et Al. 20 I 3, 5)

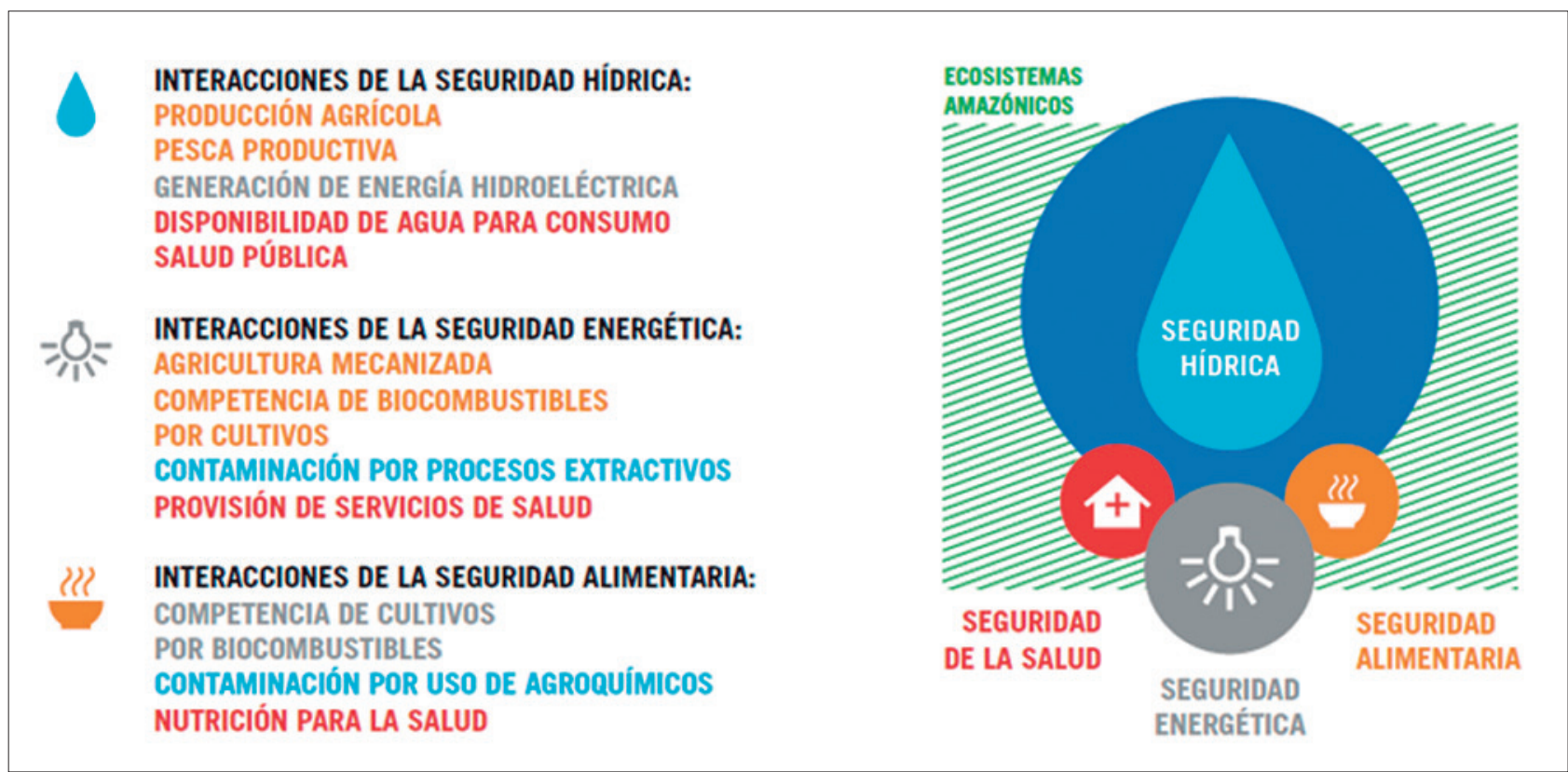

Cuadro 3: La eConomía de la eXPortación de la Amazonía (Mardas et al. 20i3, 7)

BOLIVIA

Contribución de La amazonia a L Producción nacional

Q 39\% oe uenergía hidroeléctrica

S. 41\% del ganado bovino en los

3 DEPTOS. DE BENI Y PANDO

(D) $24 \%$ de gas natural de los DEPTOS. DE COCHABAMBA Y SANTA CRUZ

INGRESOS DE EXPORTACIONES IITERNACIONRLES QUE DEPENDEN DE LA MURZZOHIA

5 US\$940 MILLONES POR SOYR, 2012

() US\$3.800 MILLONES POR GaS MaturaL, 2011

COLOMBIA

contribucion de L amazonia a La Producción macional

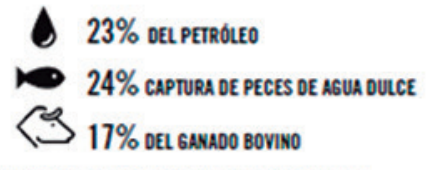

INGRESOS DE EXPORTACIONES IITERMACIONULES PROVEMIENTES DE LA AMUZONIA

US\$94 MILLONES POR PETROLEO DEL DEPTO. DE PUTUMAYO, 2000

\section{BRASIL}

Contribucion de u amazonia a L Producción macional

(1) $17 \%$ oe gas natural oel estado de amazonas

Q $11 \%$ oe u energia hlorocetétrica

(3) $37 \%$ оеL gанао войо

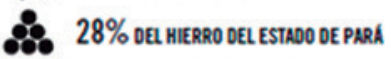

ingresos oe Exportaciones IITteruacionules provemiemtes DE LA NUZZOMLA LEGH

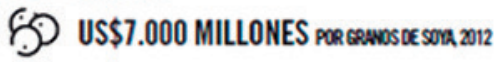
SS US\$1.600 MILLONES POR CARNE BONNA 2012
(9) US\$500 MILLONES POR Madera, 2012
80 US\$8.800 MILLONES POR MINERAL DE

ECUADOR

CONTRIBUCION de U andzonia a L PRoducción macional
Q 35\% de u ENergía hidROELÉctrica
( $99 \%$ del petróleo
IMGRESOS OE EXPORTACIONES IMTERMACIONRLES PROVENIERTES DE L AMUZONIA
US\$8.900 MILLONES POR PETROLEO, 2010 (53.800 MILUNES PRODUCCIÓN POR PETRÓLEO CRUDO Y GAS NATURAL)

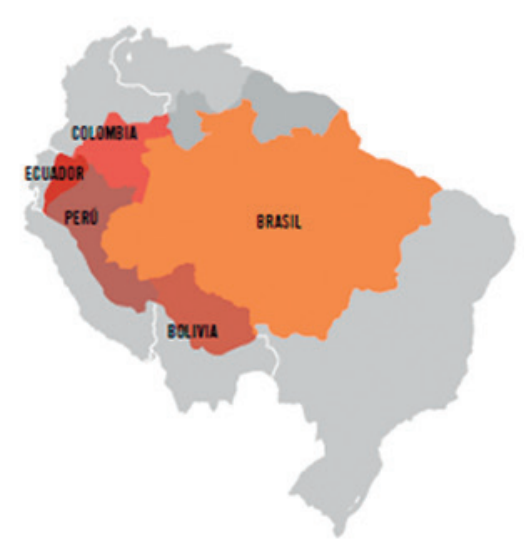

PERÚ

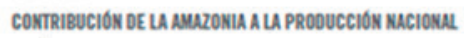

(.) $73 \%$ del petróleo Y gas natural Líquido

Q 22\% oe la energíahidroelétrica

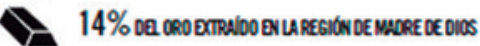

(1) US\$23.000 MILLONES de u punta de gas

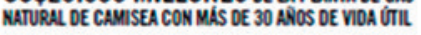

INGRESOS OE EXPORTACIONES IITERRUCIONALES PROVEMIENTES DE LA AMUZOMIA
5 US\$196 MILLIONES POR CAFE DE LOS
(9) US\$166 MILLIONES POR MaOERa, 2011 
en la región amazónica cuenta con accesos a agua tratada (Mardas et al., 2013). Sin embargo se prevé que la deforestación a gran escala reducirá las lluvias hasta en un 21\% para el 2050 (Mardas et al., 2013: 9).

La minería, la extracción petrolera, la generación de energía, la agricultura mecanizada, son actividades con grades requerimiento de agua y energía (estrechamente relacionadas en la región con la energía hidroeléctrica), es decir que dependen que la seguridad hídrica. (Mardas et al., 2013: 7).

El valor estimado de los servicios ecosistémicos — ahora mediáticos — de la Amazonía para la región, está en el rango de las decenas de miles de millones de dólares por año, como se puede ver también en el cuadro 3.

Y los planes nacionales apuntan hacia un desarrollo acelerado en la región. Por ejemplo, se prevé construir 30 represas nuevas en la Amazonía brasileña para el 2020 y 29 en la Amazonía andina. Específicamente en el caso de la Amazonía peruana, actualmente sólo se explota menos del 1\% del potencial técnico, además, el crecimiento exponencial de Brasil lo ha llevado a firmar convenios bilaterales con Bolivia para gas y con Perú para energía hidroeléctrica (Mardas et al., 2013: 6).

Ahora, decir que los bosques son importantes porque constituyen sumideros significativos de carbono, porque constituyen diversos y ricos ecosistemas, o porque son proveedores de servicios ecosistémicos básicos como la regulación hídrica, etc., es cristalizar a medias, la magnitud de su importancia, ya que estos ecosistemas también son la fuente del sustento directo para sus poblaciones rurales, así como son proveedores de espacios para la vivienda, el turismo y la recreación; son el medio amiente de muchas culturas, y por lo tanto fuente de valores sociales, culturales y espirituales.

Con respecto a las poblaciones amazónicas, se estima que alrededor del $60 \%$ de los habitantes de la Amazonía boliviana, el $37 \%$ de la ecuatoriana, el 17\% de la brasilera (Mardas et al., 2013: 8), y el 62,3\% de la peruana son pobres. Específicamente en Perú, de esa población rural amazónica, el $24,6 \%$ se encuentra en pobreza extrema (INEI, 2012, citado en Minam, 2014), mientras que de los pueblos indígenas, cuyo $80 \%$ reside en zonas rurales, el $81 \%$ es pobre total y el $40 \%$ pobre extremo (INEI y Unicef 2010, citado en Minam, 2014).

\section{La gestión nacional de los bosques amazónicos}

Alrededor del 60\% de nuestro territorio nacional es Amazonía y el 15\% del área de los bosques tropicales y subtropicales se encuentra dentro de territorios indígenas (Minam y Coop. Alemana, 2014).

Con respecto a la gestión estatal de bosques y cambio climático, a pesar de que existen distintos espacios dentro del aparato estatal (Sistema Nacional de Información Ambiental, Instituto Nacional de Recursos Naturales, Organismo de Supervisión de los cursos Forestales y de Fauna Silvestre, Servicio Nacional Forestal y de Fauna Silvestre, etc.), así como diversos programas y proyectos (Estrategia Nacional de Cambio Climático, Programa Nacional del Cambio Climático, Programa Nacional de Conservación de Bosques para la Mitigación del Cambio Climático, Proyecto de Planificación ante el cambio Climático, etc.); y de que en la última década se ha avanzado en materia de gestión ambiental, de que por fin conceptos como «ministerio del ambiente» y «cambio climático» están en la boca de políticos y son de conocimiento público (tal como lo reafirma un informe del 2013 del encuentro Interclima ${ }^{1}$ ), estos diversos instrumentos, instancias estatales y proyectos que orientan la gestión del cambio climático en el Perú en su mayoría no se encuentran plenamente implementados, o no están plenamente respaldados por todos los sectores del Estado, es decir la gestión es desarticulada, propio del carácter sectorial de la gestión pública nacional en general.

Una muestra de la contradicción y desarticulación en la gestión ambiental, ha sido la emisión de leyes conocidas localmente como "paquetazos ambientales». La ley $\mathrm{N}^{\circ} 30230$ emitida en julio del 2014, llamada «segundo paquetazo ambiental», la cual le valió a Perú el "premio» del fósil de día de la organización Climate Action Network (CAN) durante la COP20 por su «su incongruencia» con las políticas ambientales. Por ejemplo, en el artículo 24 de dicha ley, se cambia la figura de los estándares de calidad ambiental y de los límites máximos permisibles, disponiendo que a partir de la vigencia de la ley se definan en términos no sólo sociales, ambientales y sanitarios, sino también que estén condicionados a análisis económicos; además, reduce en cincuenta por ciento las tarifas de las multas

1 Interclima: espacio, encuentro de diferentes sectores del estado con organizaciones internacionales y civiles para abordar temas de la gestión ambiental en elpaís. 
ambientales y las coloca a estas como último recurso usado para disuadir a las empresas (al menos por un periodo de tres años desde la emisión de la ley); así como también debilita los conceptos de «Zonificación Económica Ecológica» y el de "Ordenamiento Territorial» al determinar que ninguno de ellos «asignan usos ni exclusiones de uso» es decir, que les da un peso explícito de referenciales. En resumen, es una ley que debilita la institucionalidad y la normativa ambiental.

Otra muestra de esto, es el concepto oficial/ técnico, para usos estatales, de «ordenamiento territorial». Tal y como está planteado, no considera en su marco legal, a la mitigación del cambio climático como una variable para la planificación del territorio, por lo que el ordenamiento territorial, sólo concebiría la problemática del cambio climático en la medida en que las autoridades regionales y locales lo empleen como una herramienta de adaptación a él (Minam, 2013); es decir, en otras palabras, la manera como está estructurada legalmente la herramienta del concepto de Ordenamiento Territorial, sólo prevé la gestión del riesgo del cambio climático (adaptación al cambio climático: gestión post asignación territorial), más no la gestión de la mitigación del cambio climático (gestión pre asignación territorial). Lo cual es preocupante si se considera que la mayor cantidad de emisión de GEI del país es por USCUSS, es decir, un sector que depende directamente de la planificación y del ordenamiento territorial.

Por otro lado, tenemos a REDD+ Indígena, que es una versión adaptada, interculturalmente, de REDD+ ${ }^{2}$. En su adaptación, REDD+ Indígena se acomoda a las características de las comunidades para la gestión y monitoreo de los bosques ya que permite trabajar con pequeños capitales, algo que sería inviable con el REDD+ convencional. Es una propuesta que busca no solo la conservación de los bosques sino también la supervivencia de las comunidades nativas bajo su propia cosmovisión, busca la efectiva trasferencia de la gobernanza ambiental a las bases, sin la cual los beneficios de REDD+ no podrían ser equitativamente distribuidos.

Pero a pesar de que se le considera a REDD+ Indígena una adaptación intercultural con salvaguardas

2 Sistema internacional de reducción de gases de efecto invernadero causados de la degradación y deforestación de los bosques. Mecanismo de mitigación del cambio climático y compensación, desarrollado bajo la CMNUCC que busca reconocer y proveer incentivos positivos a los países en vías de desarrollo para proteger sus recursos forestales, mejorar su gestión y utilizarlos de manera sostenible. sociales, etc. los pueblos indígenas sostienen que las leyes nacionales sobre bosques, protección de la vida silvestre y sobre propiedad de la tierra, no cumplen con las obligaciones internacionales en materia de derechos indígenas, y que les recortan el margen de acción para cumplir con las metas planteadas por REDD+ Indígena. Lo cual es cierto, y se puede ver a simple vista en la problemática nacional del reconocimiento y titulación de comunidades nativas, en la cual hay una larga fila de espera de decenas de comunidades nativas esperando ser definitivamente demarcadas y tituladas. El informe REDD+ Indígena (Minam y Coop. Alemana, 2014), considera, con respecto a la propiedad de la tierra que: «...el tema es considerado alarmante y difícil de abordar en la negociaciones. Territorios indígenas sin reconocer pueden ser objetos de apropiación para proyectos REDD+ y esta es una preocupación compartida ya que podría deslegitimar todo el esquema comercial que se propone». REDD+ Indígena también tiene la falencia de no contar de manera plena con un sistema de monitoreo y supervisión.

Finalmente, algo que también resalta en la gestión ambiental estatal y que se mencionó en el balance de Interclima 2013, es la necesidad de capacitar a los funcionarios de los distintos niveles (nacional, regional, local) del gobierno para nivelar sus capacidades de gestión del cambio climático; e incrementar el presupuesto para su investigación.

\section{La deforestación}

El tamaño del mercado negro forestal en el país es monstruoso. La tasa de ilegalidad del sector maderero es de al menos el 80\% de su producción (Uceda, 2016, "El inspector talado», La República, 26 de enero), por lo cual se pierde aproximadamente 220 millones de dólares (Minam, 2013; citado en Minam, 2014). $Y$ al ser un sector informal al minoreo, tiene una importancia mucho mayor para los medios de vida rurales y las economías locales (Cordero, 2011:7).

Por otro lado, la deforestación y a raíz de ella, el posterior cambio de uso de suelo, es la principal fuente de emisiones de GEI en el Perú, equivalente al $41 \%$ del total emanado (Minam y BID, s.f.). La deforestación, a pesar de ser el sector que da mayores emisiones de GEI, también es el sector de mayor incertidumbre debido a la falta de información georeferenciada histórica sobre el cambio de uso de la tierra. Se estima que en un escenario 
$\mathrm{BAU}^{3}$, las emisiones netas de GEI (emisiones menos capturas de CO2) en el año 2030 se incrementaran en un $56.21 \%$ con respecto a la línea de base de las emisiones del 2010 (Gobierno del Perú, 2015).

El sector maderero ilegal funciona con exportaciones de madera blanqueada, es decir, empresas madereras con concesiones otorgadas por el Estado, comunidades o individuos habilitados para talar, usan sus áreas asignadas como pantalla para justificar la venta de madera de bosques externos a su concesión, de bosques no autorizados. Está avalado por una red criminal y de corrupción que abarca desde parlamentarios y funcionarios del poder judicial, pasando por funcionarios de los gobiernos regionales e instituciones fiscalizadores del Estado hasta grupos organizados de civiles.

Un periodista de investigación (Luna, diario $\mathrm{La}$ República, 3 de dic. del 2015), lo expresa así: «El tráfico de madera es una de las actividades criminales más rentables del Perú junto con la minería ilegal de oro y el narcotráfico... desde que en el 2009 se implementó el Tratado de Libre Comercio con EE.UU., hasta el 2013, Osinfor ${ }^{4}$ ha constatado que con documentos oficiales se ha movilizado ilegalmente cantidades de madera estimadas en 134 millones de dólares. Estos cargamentos exportados tenían documentos formales que los avalaban, pero con información falsa.»

El panorama de la deforestación en el Perú tiene varias causales (Cardoso, 2011), (Che Piu y Menton, 2013, citado en Gobierno del Perú, 2014: 4), (Minam, 2014), (Gobierno del Perú, 2015):

- La poca accesibilidad del costo de los planes de manejo forestal.

- La poca diversificación productiva en la región de la cuenca amazónica.

- La globalización, el aumento de la demanda de productos agrícolas y extractivos y las posteriores políticas públicas de industrialización.

- La expansión de las fronteras agrícolas y ganaderas a pequeña escala por los colonos (la migración desde la sierra hacia la selva) y las familias desplazadas — que talan el bosque para desarrollar agricultura de subsistencia $y$ en algunos casos con fines comercialesaproximadamente el 88\% del área deforestada

3 «Business as usual» o en español «negocios como siempre»: proyección en un contexto en que se continúe conelmodelodedesarrollocomoelact ual,sinhacertomarmedidasdemitigaciónalcambioclimático.

4 Organismo de Supervisión de los Recursos Forestales y de FaunaSilvestre. pasa a convertirse en terreno agrícola, mientras que el aproximadamente el 10\% del área pasa a ser usado como pasturas.

- La falta de claridad en la tenencia de la tierra: concesiones madereras que se superponen a territorios indígenas y a áreas naturales protegidas.

- La pobre fiscalización de políticas y normas de los sectores involucrados.

- La corrupción de las autoridades estatales: a 9 regiones del país le fueron transferidas las competencias forestales en el 2010, con esta medida, los funcionarios regionales (muchos de ellos, dueños de madereras), quedan encargados de las supervisiones forestales. Solo en la región de Ucayali, decenas de comunidades indígenas enfrentan conflictos con madereras cuyas concesiones se superponen a las de sus territorios, y el estado no termina el proceso de delimitación y titulación de las tierras indígenas.

De continuar la deforestación, el cambio climático no hará más que agravar la situación de sus ecosistemas, lo cual disminuirá las lluvias y la escorrentía tanto dentro como fuera de la cuenca, afectando el ciclo del carbono mundial (IPCC, 1997).

\section{Perspectivas y recomendaciones para la gestión de los bosques en el contexto de cambio climático}

La conservación de los bosques amazónicos peruanos, pasa inevitablemente por desarmar el sistema ilegal de deforestación e instalar un sistema de aprovechamiento forestal sostenible social, económica y ambientalmente, para lo cual a su vez debe darse la batalla en los siguientes aspectos/campos de este complejo cuadro:

\section{Cuestiones transversales}

Visiones de gobierno a cambiar: "beneficio a corto plazo", sectorialización y corrupción

El paradigma desarrollista-productivista que guía desde hace décadas a las políticas públicas, no cuenta con profundas consideraciones ambientales ni de largo plazo. Insistir en darle prioridad al crecimiento del PBI como solución a todos nuestros males, es iluso pues la 
pobreza en la región no tiene causas coyunturales sino estructurales, por lo tanto no se desvanecerá con sólo el aumento del ingreso monetario, sino que se debe reformar los medios naturales, infraestructurales y sociales para la reversión de dicha situación (Sejenovich, 2015). En la región, la relación entre pobreza, crisis ambiental y acumulación a corto plazo, presenta una particular complejidad: que es que la problemática ambiental, forma parte constitutiva del problema mismo del carácter desigual y dependiente del modelo económico (Sejenovich, 2015).

«Sectoralismo», es una característica común a los países de la región, con este concepto nos referimos a diseñar y aplicar leyes, planes, programas y proyectos nacionales sin una visión integral, en donde cada sector del estado o ministerio trabaja sin coordinación ni acuerdo con sus homólogos, en donde prácticamente no hay objetivos ni planes de trabajo comunes. Para Sejenovich (2015), la visión sectoralista privilegia la producción y la eficiencia de corto plazo, minimiza la importancia de las interacciones, y en general, otorga poco espacio para la planificación, para la participación y para la articulación con la ciencia, la tecnología y con la calidad de vida de la población.

La corrupción estatal, es probablemente el obstáculo más grande a superar en el objetivo de conservar los bosques peruanos. Esta se puede ver claramente en casos como el del pasado vicepresidente de la región amazónica de Ucayali que a su vez es dueño del Grupo Henderson, que cuenta con una empresa maderera que es titular de una concesión estatal y que ha sido auditada en el año 2013 por Osinfor debido al blanqueamiento de 4,491, 892 metros cúbicos de madera.

Según la Defensoría del Pueblo (2015), cotidianamente se pone en evidencia la insuficiencia en la representación política, así como de las vías jurisdiccionales y administrativas para resolver los conflictos sociales y garantizar un escenario estable para la vigencia de los derechos, las inversiones y la protección del medio ambiente. El caso más emblemático para este análisis probablemente sea el caso del líder asháninca, Edwin Chota, asesinato en el año 2014, por la mafia maderera luego de denunciar ante las autoridades estatales, durante meses, a los madereros ilegales que rondaban su comunidad sin titular.

Es así como un cambio de visión, de lo señalado líneas arriba, es la llave para habilitar medidas que den paso a alcanzar el objetivo de conservación. Que serían las siguientes:

\section{Condiciones habilitantes}

Con «condiciones habilitantes» se entiende aquellas medidas legales, normativas y organizacionales del aparato estatal que garanticen/habiliten el camino de los diversos proyectos que puedan haber para la conservación y la gestión sostenible de los bosques.

El Plan Nacional de Cambio Climático contempla 15 opciones de mitigación (Gobierno del Perú, 2014), pero no cuenta con condiciones habilitantes como por ejemplo: una normativa que reduzca los altos costos de transacción de los sistemas de pago por servicios ecosistémicos, incentivos económicos, capacitaciones para los usuarios de los bosques, una normativa clara, simple y unificada de la títulos de propiedad de los bosques y suelos, un sistema eficaz de vigilancia y control de la tala ilegal, un sistema integral y actualizado de información forestal y un efectivo sistema de ordenamiento territorial (Minam, 2014).

La ausencia de estas condiciones habilitantes le restan rentabilidad y competitividad a la preservación y la gestión sostenible de los bosques y favorecen el mercado de la tala ilegal.

\section{Reforma del sistema de concesiones forestales}

Como ya se ha explicado líneas arriba, el sistema de la tala ilegal que mueve millones de soles en el país se sustenta en el mecanismo del blanqueamiento de madera y la mayor cantidad de madera blanqueada proviene de las concesiones forestales que son las que tienen más mayor facilidad para blanquear grandes cantidades.

$\mathrm{Si}$ bien Osinfor tiene ya una función de vigilancia sobre las concesiones y exportaciones de madera, necesita autonomía y reforzar sus sistemas de información para hacer de manera más precisa e implacable esta tarea. Al respecto, también sería urgente endurecer las medidas coercitivas a través de fuertes multas y el retiro definitivo de las licencias de concesión.

\section{Delimitación, titulación de tierras y sistema de catastro unificado y georeferenciado}

En términos absolutos, las áreas anuales de deforestación ocurren principalmente en bosques sin derechos, predios privados, comunidades nativas y bosques no concesionados, lo cual sugiere que la falta de asignación 
de derechos es uno de los factores principales de deforestación y degradación (Minam,2014).

El experto, Bastian (2011), que ha analizado el caso de la deforestación en la Amazonía brasilera, cuya realidad es bastante parecida a la que atraviesa la Amazonía peruana, concluye que una contribución significativa, "perenne», de largo plazo y sostenible a través de los cambios de gobierno, es ordenar la tenencia de la tierra: «asumir la efectiva gobernanza sobre la tenencia de la tierra». Dentro de su análisis, ve que hay dos variables que no se suelen tomar en cuenta para en el complejo cuadro de la deforestación amazónica: 1) la especulación del valor de las tierras (es decir, la expectativa de que habrá demanda por la tierra) y 2) la ausencia de gobernanza de la tierra o lo que sería un sistema efectivo sobre la titulación, asignación y concesión de tierras y una falta de ordenamiento territorial.

Existen dos características de la Amazonía brasileña que también tiene la Amazonía peruana (Bastian, 2011): la adquisición informal y posterior deforestación de tierras, sobre todo públicas, que es altamente rentable. $\mathrm{Y}$ esta dinámica de adquisición informal no puede parar sin gobernanza de la tierra $y$ sin un marco legal efectivo, sin un reconocimiento y empoderamiento de los propietarios. Tener una buena gobernanza de la tierra, un catastro moderno, permitiría:

- Establecer con seguridad políticas relacionadas a: reforma agraria, crédito agrario, tributación sobre la tierra.

- Dificultar la posesión privada inapropiada.

- Reglar los procesos de compra de tierras, para limitar el monopolio de tierras, etc.

- Hacer la zonificación de uso de la tierra, etc.

- Limitaría la especulación por tierras.

- Afianzaría el mecanismo REDD+ Indígena.

Entonces, es necesario legislar desde el Estado sobre la tenencia de la tierra: aclarar fronteras entre comunidades, concesiones y áreas naturales protegidas, terminar de titular a las comunidades campesinas y nativas, no sólo reducirá el número de conflictos socio ambientales sino que es una condición habilitante básica que generaría seguridad sobre los actores sociales que pretendan invertir en proyectos de bosques sostenibles y otros; además que simplificaría los procesos de vigilancia y supervisión de la Osinfor.

\section{Ordenamiento territorial}

Es un error de perspectiva separar adaptación al cambio climático de mitigación al cambio climático: el cambio climático ya empezó, ya está aquí y mitigarlo es una manera de adaptarse a él.

Tal y como está formulado por el Estado, el concepto de «ordenamiento territorial» sólo prevé la gestión del riesgo del cambio climático, es decir la adaptación al cambio climático, más no la gestión de la mitigación del cambio climático. Lo cual es preocupante si se considera que la mayor cantidad de emisiones de CO2 del país proviene de USCUSS, es decir, un sector que depende directamente de la planificación y el ordenamiento territorial. La mitigación en el sector USCUSS está íntimamente ligada al manejo territorial: manejo forestal sostenible, sistemas agroforestales, consolidación de áreas naturales y reforestación.

\section{Empoderar a los usuarios del bosque}

Un factor en contra para el manejo forestal sostenible es la limitada capacidad organizativa y de gobernanza de las organizaciones locales ligadas a los bosques (Cordero, 2011: 20).

Empoderar a las poblaciones locales amazónicas, a las comunidades nativas, y a las comunidades campesinas, a la sociedad civil nacional en general, es la mejor garantía de vigilancia y protección de los bosques. Pero empoderarlos no pasa solo por capacitarlos sino primero por el simple hecho de reconocer sus derechos sobre los bosques y la tierra, y antes de capacitarlos, por facilitarles el acceso a información que les permita sentir la necesidad de empoderarse, de mitigar y adaptarse al cambio climático.

\section{Expandir y perfeccionar los sistemas de información}

Para expertos en el tema, otra cosa que se requiere con urgencia es expandir y perfeccionar los sistemas de información, por ejemplo Mardas et al. (2013) detalla que lo que se requiere es diseñar indicadores ambientales, económicos y sociales sobre las seguridades: hídricas, alimentaria, energética y de la salud; mapeos de zonas de alto riesgo para estas seguridades, mapeo de vulnerabilidades de los distintos sectores y poblaciones amazónicas, un sistema de alerta temprana que aproveche el mapeo 
de zonas de alto riesgo y que se enfoque en los fenómenos climáticos extremos, cambio en el uso de la tierra y focos de contaminación que afecten las seguridades.

Toda información será recabada al respecto serviría para el correcto funcionamiento de los sistemas de supervisión, control y vigilancia.

\section{Supervisión, control y vigilancia}

Se requieren sistemas de vigilancia, control y supervisión para programas como REDD+ Indígena —que aún no lo tiene implementado como se debe-, las concesiones forestales y general cualquier proyecto forestal que se pretenda emprender en el país.

\section{Otras aristas sobre las cuales trabajar}

\section{El lugar mediático de los casos ambientales}

Las noticias ambientales no se caracterizan por tener un lugar mediático privilegiado en los medios peruanos. La deforestación de los bosques es una problemática que no llama la atención de la opinión pública, probablemente debido a la escasa y prácticamente nula educación ambiental.

Acá el rol de los medios principales de comunicación es básico para empoderar a las poblaciones y con esto generar una presión sobre las políticas públicas estatales.

\section{Trabajar en conjunto con los demás países de la cuenca amazónica}

Con respecto las instancias estatales, se requiere terminar de implementar, consolidar y financiar a las Autoridades Regionales Ambientales para que terminen aplicando políticas territoriales integrales y contundentes.

Mardas et al. (2013) propone que se creen grupos de trabajo y cooperación regionales, para conservar la Amazonía y mantener las seguridades. Lo que se llaman «grupos nexus» conformados por expertos sénior de diferentes ministerios para intercambiar información, definir prioridades e identificar vacíos en las políticas, que podrían tomar como modelos los «Equipos de Trabajo Presidencial» como la UKP4 de Indonesia.
Trabajar de la mano con las comunidades campesinas y pueblos indigenas

Luego de empoderar vendría el diálogo intercultural entre las comunidades locales y el estado acerca del ordenamiento territorial, la zonificación económico ecológica y la manera de gestionar sustentablemente los bosques.

Una iniciativa que ya tiene ańos de implementada y que está dando buenos resultados pero que aún falta replicarse considerablemente es la de REDD+ indígena. Otra alternativa podría ser seguir el ejemplo de gestión nacional de los bosques con acompańamiento de las poblaciones indígenas que ha implementado Bolivia, a través de la Mesa Indígena del Bosque y el Cambio Climático, conformada por la Confederación de Pueblos Indígenas de Bolivia (CIDOB), estado y organismos de cooperación internacional, que tiene como fin integrar los métodos occidentales de adaptación con los saberes tradicionales de estos pueblos (Feldt, 2011).

Trabajar de la mano con las comunidades no sólo es lo más democrático, sino también es bastante acertado y estratégico considerándose que serán ellos los ejecutores directos de los proyectos y las políticas y la sociedad civil más cercana e involucrada en la problemática.

\section{Educación ambiental}

Dos estudios realizadosa nivelde Lima Metropolitana, en el 2009 y en el 2012, revelan que la población tiene conocimientos muy elementales acerca del cambio climático, sus impactos y sus maneras y la manera de enfrentarlo, que no consideran al cambio climático como un tema prioritario y solo el $4,8 \%$ consideraba al cambio climático como uno de los tres temas más preocupantes del país (MinamInterclima, 2013).

Para tener una sociedad civil activa que presione a los gobiernos a conservar los bosques, esa sociedad civil primero debe tener conocimiento de la problemática de la deforestación en el país y de los efectos de esta sobre diversos aspectos de importancia nacional y sobre el cambio climático. 


\section{Mejorar la capacidad de diálogo y de tratamiento de conflictos del Estado}

En palabras del informe N. ${ }^{\circ} 156$ de la Defensoría del Pueblo" "cuando las causas inmediatas se montan sobre las estructurales, los conflictos pueden ser muy violentos, de larga duración y de una gran cohesión social. Estos son los elementos que preceden a movimientos políticos de mayo aliento, especialmente en contextos de identidades culturales fuertes y cada vez más conscientes de su exclusión. Un ejemplo de esta situación es perceptible en los movimientos sociales indígenas de la Amazonía peruana y de ciertas zonas de la sierra sur.»

En el año 2011 el 47.7\% del total de los conflictos registrados por dicha institución fueron de índole socioambiental (Defensoría del Pueblo, 2012).

Según la Defensoría del Pueblo (2012), el Estado no ha cumplido su función de regulación y de control en toda su extensión y ha permitido que los problemas sean abordados asimétricamente por los poderes fácticos, sociales y de mercado. Algo que también se menciona en dicho informe es que la cultura del diálogo es incipiente, y que los valores como la tolerancia, el respeto a la ley y el reconocimiento del otro no son parte de la idiosincrasia o prácticas de los actores estatales ni de los actores sociales en general. Además, y algo muy importante, observa la persistencia de «falsos diálogos» — como los llama— que se presentarían en las siguientes formas: i) el uso del diálogo como una estrategia para ganar tiempo y consolidar la posición de la fuerza; ii) la práctica del diálogo sin convicción y sin arte en manos de un Estado que suele llegar a dialogar después de la violencia; iii) un diálogo desigual que atrae a una de las partes hacia un terreno disparejo, en el que sus recursos informativos,económicos y políticos no serán suficientes para una negociación equilibrada; iv) el ejercicio del diálogo, en contextos de presión $\mathrm{y}$ violencia, que interfiere en la racionalidad de las decisiones y v) el diálogo que concluye con un acta que no se cumple (Luque, 2009, citado en Informe de la Defensoría del Pueblo $N^{\circ} 156$ ). Con estos antecedentes es entendible la existencia de desconfianza por parte de la población hacia autoridades como el Estado. Hay mucho que avanzar en este aspecto.

5 Órgano gubernamental autónomo que tiene como una de sus funciones seguir las dinámicas de la conflictividad a nivel nacional.

\section{Conclusiones}

Se suele ver a la gestión del riesgo del cambio climático, como sinónimo de adaptación cuando en realidad, para el caso particular peruano, la mitigación, cristalizada concretamente en la reducción de la deforestación, es una manera de gestionar el riesgo del cambio climático: como se ha podido ver líneas arriba, los bosques saludables son la base de la seguridad hídrica que a su vez es la base de otras seguridades para el país y la región.

Se requiere un cambio de visión país, y este cambio pasa por reconocer la magnitud de la importancia de la Amazonía, así como también pasa por ver al cambio climático como un multiplicador exponencial de amenazas y un escenario nada remoto.

\section{Referencias}

Bastian, Philip (2011). La deforestación de la selva amazónica: causas y soluciones, en Politica ambiental. Economía verde: desafios y oportunidades. [en línea]. Consultado el 31 de marzo del 2016, http://www.ie.ufrj.br/images/gema/ Gema_Artigos/2011/PoliticaAmbiental08portugues.pdf

Cambia.pe, Perú recibe el «Fósil del día» por el "paquetazo ambiental», consultado el 21 de marzo del 2016 en http://cambia.pe/ peru-recibe-el-fosil-del-dia-por-el-paquetazo-ambiental/

Cordero, D. (2011). Los bosques en América Latina [en línea]. Consultado el 31 de marzo del 2016, http:// library.fes.de/pdf-files/bueros/quito/08364.pdf

Defensoría del Pueblo (2012). Violencia en los conflictos. Informe Defensorial $N^{\circ} 156$ [en línea]. Consultado el 30 de marzo del 2016, http://www.defensoria.gob.pe/modules/ Downloads/informes/defensoriales/informe-156.pdf

Defensoría del Pueblo (2015). Décimo Octavo Informe Anual de la Defensoría del Pueblo enero-diciembre 2014 [en línea]. Consultado el 30 de marzo del 2016, http:// www.defensoria.gob.pe/modules/Downloads/informes/ anuales/Decimoctavo-Informe-Anual.pdf

Diario La República (2015). «Madereros de Ucayali protestan contra la erradicación de la tala ilegal» [en línea] $L a$ República, 14 de octubre, consultado: 12 de marzo del 2016, http://larepublica.pe/politica/710427-madererosde-ucayali-protestan-contra-la-erradicacion-de-la-talailegal

Feldt, Heidi (2011). Fortalecimiento de la Organizaciones Indigenas en América Latina: Pueblos Indigenas y Cambio 
Climático [en línea]. Consultado el 31 de marzo del 2016, http://www.cop20.pe/ck/fortalecimiento-deorganizaciones-indigenas-en-america-latina-pueblosindigenas-y-cambio-climatico/

Gobierno del Perú (2014). Bosques y cambio climático [en línea]. Consultado el 31 de marzo del 2016, http://www.cop20.pe/caja-de-herramientas/ presentaciones-de-la-cop20/

Gobierno del Perú (s.f.). Anexo: Supuestos y metodologías contempladas como parte de las contribuciones nacionales en mitigación [en línea]. Consultado el 30 de marzo del 2016, http://www.minam.gob.pe/indcs/wpcontent/uploads/sites/100/2015/06/Supuestos-yMetodolog\%C3\%ADas-contempladas-como-parte-delas-Contribuciones-Nacionales-en-Mitigaci\%C3\%B3n. pdf

GRUPO INTERGUBERNAMENTAL DE EXPERTOS SOBRE EL CAMBiO CLIMÁtico (IPCC) (1997). Informe especial del Grupo de Trabajo II del IPCC, Impactos regionales del cambio climático: Evaluación de la vulnerabilidad. Resumen para responsables de políticas [en línea]. Consultado el 30 de marzo del 2016, https://www.ipcc.ch/pdf/specialreports/spm/region-sp.pdf

JimÉNEZ y URRUNAGA (2014). «Vicepresidente de Ucayali blanquea madera de la tala ilegal, según Osinfor» [en línea] La República, 2 de octubre, consultado: 31 de marzo del 2016, http://larepublica.pe/02-10-2014/ vicepresidente-de-ucayali-blanquea-madera-de-la-talailegal-segun-osinfor

Mardas, N. et al. (2013). Agenda de seguridad para la Amazonía: Resumen de hallazgos y recomendaciones iniciales. Global Canopy Programme y International Center for Tropical Agriculture [en línea]. Consultado el 30 de marzo del 2016, file:///C:/Users/PERSONAL/ Downloads/Agenda\%20de\%20Seguridad\%20para\%20 la\%20 Amazonia.pdf

Ministerio del Ambiente (2013). 2012, Avances, retos, prioridades y orientaciones para la gestión del riesgo climático en el Perú [en línea]. Consultado el 30 de marzo del 2016, http://www.minam.gob.pe/cambioclimatico/ wp-content/uploads/sites/11/2013/10/interclima-2012. pdf
Ministerio del Ambiente (MINAM) (2014). Documento de Trabajo: Instrumentos financieros para la conservación de bosques en los gobiernos regionales amazónicos [en línea]. Consultado el 30 de marzo del 2016, http:// www.bosques.gob.pe/archivo/files/pdf/instrumentos_ financiamiento.pdf

Ministerio del Ambiente (2014). Orientaciones básicas sobre ordenamiento territorial en el Perú [en línea]. Consultado el 31 de marzo del 2016, http://www. minam.gob.pe/ordenamientoterritorial/wp-content/ uploads/sites/18/2013/10/Orientaciones-basicas-OT. pdf

Ministerio del Ambiente (2014). Sistema Nacional de Información Ambiental: Cifras Ambientales 2014 [en línea]. Consultado el 30 de marzo del 2016, http://sinia.minam. gob.pe/documentos/peru-cifras-ambientales-2014

Ministerio del Ambiente y Banco Interamericano de Desarrollo (2014). Infografia: El cambio climático en el Perú y el mundo [en línea]. Consultado el 31 de marzo del 2016, http://sinia.minam.gob.pe/novedades/ infografia-cambio-climatico-peru-mundo

Ministerio del Ambiente y Cooperación Alemana (2014). REDD+ Indigena en el Perú: Perspectivas, avances, negociaciones y desafíos desde la mirada de los actores involucrados [en línea]. Consultado el 30 de marzo del 2016, http://www.bosques.gob.pe/archivo/files/pdf/ redd_indigena_peru.pdf

LunA, N. (2015). «Operación Amazonas: La historia de la mayor incautación de madera de origen ilegal", [en línea] La República, 3 de dic. Consultado: 31 de marzo del 2016, http://larepublica.pe/impresa/ politica/723051-operacion-amazonas-la-historia-de-lamayor-incautacion-de-madera-de-origen-ilegal

República del Perú (2015). Contribución prevista y determinada a nivel nacional (INDC) [en línea]. Consultado el 30 de marzo del 2016, http://www. minam.gob.pe/wp-content/uploads/2015/09/ ESPA\%C3\%91OL.pdf

Sejenovich, Héctor (2015). La estrategia para superar la pobreza a través del desarrollo sustentable en Gobernanza Ambiental en América Latina. 